\title{
Angular response of thin-film organic solar cells with periodic metal back nanostrips
}

\author{
Wei E. I. Sha, ${ }^{1}$ Wallace C. H. Choy, ${ }^{1,3}$ and Weng Cho Chew ${ }^{1,2,4}$ \\ ${ }^{1}$ Department of Electrical and Electronic Engineering, The University of Hong Kong, Pokfulam Road, Hong Kong \\ ${ }^{2}$ Department of Electrical and Computer Engineering, University of Illinois, Urbana-Champaign, Illinois 61801, USA \\ ${ }^{3}$ e-mail: chchoy@eee.hku.hk \\ ${ }^{4}$ e-mail:wcchew@hku.hk
}

Received September 3, 2010; revised November 26, 2010; accepted December 28, 2010; posted January 13, 2011 (Doc. ID 134490); published February 4, 2011

We theoretically study the angular response of thin-film organic solar cells with periodic Au back nanostrips. In particular, the equation of the generalized Lambert's cosine law for arbitrary periodic nanostructure is formulated. We show that the periodic strip structure achieves wide-angle absorption enhancement compared with the planar nonstrip structure for both the $s$ - and $p$-polarized light, which is mainly attributed to the resonant Wood's anomalies and surface plasmon resonances, respectively. The work is important for designing and optimizing high-efficiency photovoltaic cells. (C) 2011 Optical Society of America

OCIS codes: $250.5403,050.6624,350.6050$.

Generally, unless a solar panel is mounted on an expensive tracking system, most of the time, light is incident on the array obliquely. Any improper design of the angular response [1-4] will significantly degrade the energyconversion efficiency of solar cells (SCs) due to the poor photon absorption or harvesting. For the thin-film photovoltaic cells, the thickness of the active layer must be smaller than the minority carrier or exciton diffusion length to avoid bulk recombination. Typically, the light absorption of SCs strongly depends on the incident angle [5], which is governed by Lambert's cosine law [6]. Hence, comprehensive and rigorous study of the angular response is particularly important for the optimized design of thin-film SCs. Although some work has been done on the angular response of silicon SCs [1] and thin-film organic SCs (OSCs) [3], it is highly desirable to study the angular response of periodic plasmonic SCs, which can boost the device performance. Moreover, the generalized equation of Lambert's cosine law for any periodic nanostructured SCs is important for understanding their angular response.

In this Letter, we systematically study the angular response of the thin-film OSC with the periodic metal back nanostrips. Recently, silver gratings as the front electrode have been investigated $[7,8]$. Here, we employ the periodic Au strips at the bottom of the SC, and, therefore, the blockage effect in short wavelengths can be significantly reduced. Besides, the generalized equation of Lambert's cosine law for arbitrary periodic structure is formulated. We find that the periodic strip structure can achieve wide-angle absorption enhancement compared with the (planar) nonstrip structure for both the $s$ - and $p$-polarized light. The guided complex waves (GCWs) [9] supported by the periodic strip structure contribute to the enhancement for the $s$-polarized light. The surface plasmon resonances (SPRs) excited by the subwavelength Au nanostrips contribute to the enhancement for the $p$-polarized light.

The energy conservation law in a periodic structure is given by

0146-9592/11/040478-03\$15.00/0

$$
[1-L(\theta)] \cdot \int \frac{-1}{2} \operatorname{Re}\left[\mathbf{E}_{i} \times \mathbf{H}_{i}^{*}\right] \cdot \mathrm{d} \mathbf{S}=\int n_{r} n_{i} \omega \epsilon_{0}|\mathbf{E}|^{2} \mathrm{~d} V,
$$

where $\mathbf{E}_{i}$ and $\mathbf{H}_{i}$ are the incident electric and magnetic fields, $\mathbf{S}$ is a virtual planar surface in front of the unit cell of the SC, $n_{c}=n_{r}+i n_{i}$ are the complex refractive indices of the active materials, and $L(\theta)$ is the energy loss fraction. One part of the energy loss is the scattering loss, which can be characterized by the summation of the reflectance and transmittance of the periodic structure [10]. The scattering loss can be reduced by the light guiding, enhancement, and trapping schemes. Another part of the energy loss is the metallic absorption loss, which can be reduced by engineering the metal's size, material, and position. The irradiance of the Sun is the incident power per unit area of the electromagnetic radiation at a surface, i.e.,

$$
I=\frac{-1}{2} \operatorname{Re}\left[\mathbf{E}_{i} \times \mathbf{H}_{i}^{*}\right] \cdot \mathbf{n}=\frac{\left|\mathbf{E}_{i}\right|^{2}}{2 Z} \cos \theta
$$

where $Z=\sqrt{\mu_{0} / \epsilon_{0}}$ is the wave impedance of free space and $\theta$ is the incident angle of the sunlight with respect to the normal direction of the surface $\mathbf{S}$. For the Lambertian bulk cells [1], which have the angle-independent energy loss fraction $L$, the absorption of the active layers represented by the right-hand of Eq. (1) obeys Lambert's cosine law [6] that the absorption is a cosine function of the incident angle $\theta$. It should be noted that the planar multilayered structure can be considered a special case of the periodic structure if the artificial periodic boundary conditions (PBCs) are imposed. Therefore, Eq. (1) is the generalized equation of Lambert's cosine law for any periodically structured SCs.

Figure 1(a) illustrates the typical structure of the standard OSC without any optimization. The heterojunction active layer is composed of copper phthalocyanine $(\mathrm{CuPc})$ and fullerene $\left(\mathrm{C}_{60}\right)$ as the electron donor and acceptor, respectively. The bathocuproine (BCP) layer is a 


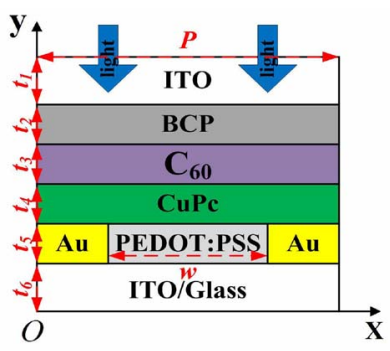

(a)

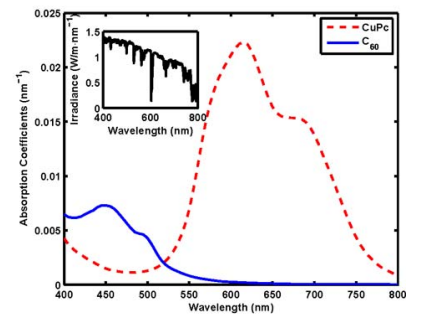

(b)
Fig. 1. (Color online) (a) Schematic structure of the unit cell of the OSC. The structural parameters are $t_{1}=100 \mathrm{~nm}$, $t_{2}=10 \mathrm{~nm}, t_{3}=40 \mathrm{~nm}, t_{4}=30 \mathrm{~nm}, t_{5}=30 \mathrm{~nm}, t_{6}=100 \mathrm{~nm}$, $w=100 \mathrm{~nm}$, and $P=200 \mathrm{~nm}$. (b) Absorption coefficients of $\mathrm{CuPc}$ and $\mathrm{C}_{60}$. The inset is the Sun irradiance spectrum of an air mass of 1.5 (AM1.5).

spacer layer for extracting electrons. The metallic back nanopattern is made from the $\mathrm{Au}$ strips and Poly (3,4-ethylenedioxythiophene):poly (4-styrenesulfonic acid) (PEDOT:PSS; AI4083) that can collect holes. We use the finite-difference frequency domain method [11] to calculate the angular response of the OSC. The perfectly matched layer and Mur-absorbing boundary conditions are used at the top and bottom boundaries of the simulation region. The PBCs are adopted at the left and right boundaries. The interfaces between different materials are treated by the one-sided difference strategy and the averaged material model. The complex dielectric constant of $\mathrm{Au}$ can be expressed by the Brendel-Bormann model [12]. The complex dielectric constants of the organic materials are measured by ellipsometry [13]. In modeling the light absorption of the SC, the Sun irradiance spectrum [as shown in the inset of Fig. 1(b)] has been considered.

Figure 1(b) shows the absorption coefficients of $\mathrm{CuPc}$ and $\mathrm{C}_{60}$. The separated absorption peaks can improve the light absorption of the OSC, which can be realized by different enhancement mechanisms with respect to different materials. Compared with the absorption of the nonstrip structure made from the planar PEDOT:PSS layer, the enhancement factors of the absorption for the strip structure of the laterally periodic Au-PEDOT: PSS-Au pattern are shown in Fig. 2 for the $s$ and $p$ polarizations, respectively.

For the $s$ polarization, the absorption enhancement is obtained from 400 to $500 \mathrm{~nm}$ with the very strong absorption peaks especially at the oblique angles. The rapid variations in the absorption over narrow frequency bands imply the resonant Wood's anomalies [9,14]. Figure 3(a) shows the $E$ field of the absorption peak pointed by the arrow in Fig. 2(a) for the Wood's anomalies. The intense $E$ field in the active layer is bounded between the periodic nanostrip pattern and the BCP layer. The $E$ field is related to the GCW supported by the periodic strip structure with the phase matching condition of $k_{0} \sin \theta+$ $2 \pi n / P= \pm \operatorname{Re}\left(\beta_{g}\right), n= \pm 1, \pm 2, \ldots$, where $P$ is the periodicity, $k_{0}$ is the incident wave vector, and $\beta_{g}$ is the propagation constant of the GCW. The condition is easy to be satisfied in the standard OSCs considering the broadband and wide-angle Sun illumination. For confirming the forced-resonance behavior of the Wood's anomalies, we calculate the averaged power density flowing in the $x$

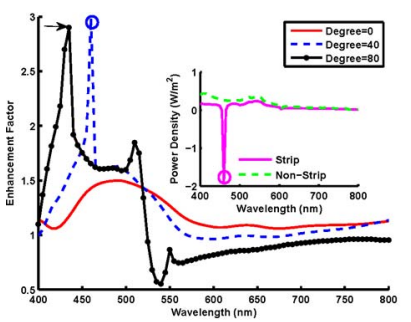

(a)

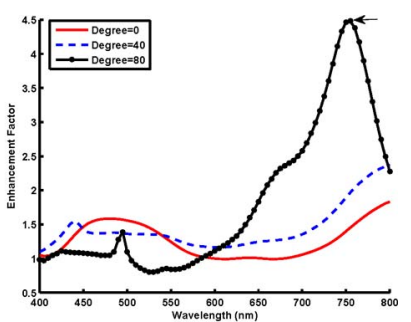

(b)
Fig. 2. (Color online) Absorption of the periodic strip structure is compared to that of the nonstrip structure, replacing the laterally periodic Au-PEDOT:PSS-Au pattern with the planar PEDOT:PSS layer. The near-field profiles of the absorption peaks pointed by the arrows are shown in Fig. 3. (a) Absorption enhancement factor for the $s$ polarization. The inset shows the averaged power density of the Au-PEDOT:PSS-Au pattern along the $x$ direction. The negative power density peak due to the opposite propagation direction coincides with the absorption peak at $\theta=40^{\circ}$ with the wavelength $460 \mathrm{~nm}$. (b) Absorption enhancement factor for the $p$ polarization.

direction for the Au-PEDOT:PSS-Au pattern (strip structure) and PEDOT:PSS layer (nonstrip structure). For the strip structure, as shown in the inset of Fig. 2(a), the negative (opposite direction) power density peak induced by the $n=-1$ space harmonics coincides with the absorption peak at $\theta=40^{\circ}$ and $460 \mathrm{~nm}$. In addition, the spectral overlap between the Wood's anomalies and the material absorption of $\mathrm{C}_{60}$ will be of a good help for short wavelength photon harvesting. Particularly, we can observe the sharp oscillations of the enhancement factor at $\theta=80^{\circ}$ from 510 to $550 \mathrm{~nm}$. The sharp oscillations result from the overlap between the waveguide mode (WM) in the nonstrip structure and the Wood's anomalies and the WM in the strip structure. It should be noted that the GCW is the eigenstate of Maxwell's equations for an arbitrary periodic structure and cannot be excited in the planar nonstrip structure by the plane wave due to the momentum mismatch $\left(\beta_{g}>k_{0}\right)$. However, the WM can be found in the planar structure [11] and can be understood by the mode coupling between the excitation solution and the eigenstate. The WM and GCW can be located by the methods in $[11,14]$, respectively.

For the $p$ polarization, the broadband absorption enhancement is obtained from 650 to $800 \mathrm{~nm}$, especially at the oblique angles. Figure 3(b) shows the $H$ field of the absorption peak pointed by the arrow in Fig. 2(b). The concentrated $H$ field at the interface between the $\mathrm{Au}$ and $\mathrm{CuPc}$ layers is due to the SPRs excited by

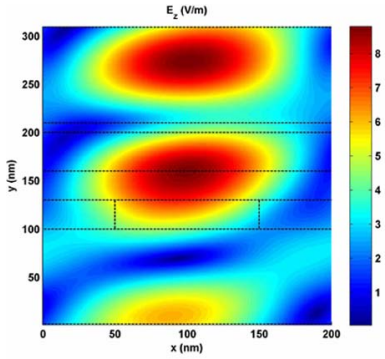

(a)

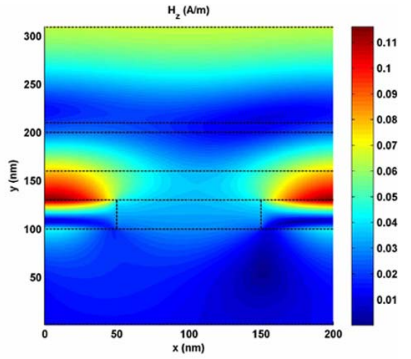

(b)
Fig. 3. (Color online) (a) $E_{z}$ field profile of the $s$ polarization at $\theta=80^{\circ}$ with $435 \mathrm{~nm}$. (b) $H_{z}$ field profile of the $p$ polarization at $\theta=80^{\circ}$ with $755 \mathrm{~nm}$. 


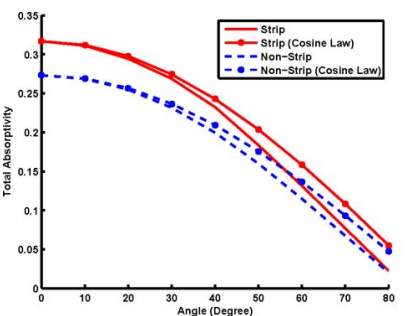

(a)

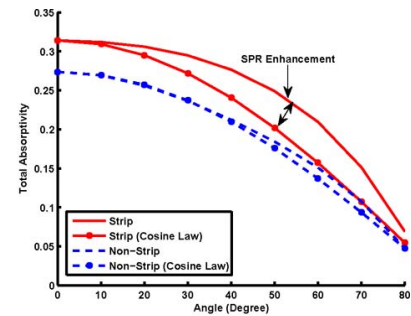

(b)
Fig. 4. (Color online) Total absorptivity as a function of the incident angle $\theta$. The generalized Lambert's cosine law (straight-dotted and dash-dotted curves) are calculated by $A_{0}^{t} \cos \theta$, where $A_{0}^{t}$ is the total absorptivity under the vertical incidence condition. (a) $s$ polarization; (b) $p$ polarization.

the evanescent waves produced by the subwavelength nanostrips. Regarding the absorption peaks around $750-800 \mathrm{~nm}$ in Fig. 2(b), they are blueshifted as the incident angle increases due to the blueshift of the plasmoncoupled WMs. Furthermore, there are two small peaks at 440 and $495 \mathrm{~nm}$ induced by the WMs. In addition, the optical path is prolonged by the back grating pattern. By studying the SPR spectrum [Fig. 2(b); straight-dotted curve] and the absorption spectrum of CuPc [Fig. 1(b); dashed curve] together, it is observed that the SPR peak at $675 \mathrm{~nm}$ is weaker than that at $755 \mathrm{~nm}$ because of the stronger absorption of $\mathrm{CuPc}$ at $675 \mathrm{~nm}$.

Figure 4 shows the total absorptivity as a function of the incident angle for the $s$ and $p$ polarizations, respectively. The ideal total absorptivity governed by the generalized Lambert's cosine law is calculated by $A_{0}^{t} \cos \theta$, where $A_{0}^{t}$ is the total absorptivity under the vertical incidence condition. The total absorptivity of the strip structure is noticeably better than that of the nonstrip structure for both polarizations. The improvements are caused by the Wood's anomalies and the SPRs that have been explained previously. When the incident angle increases, the total absorptivity for the $p$ polarization decays slower than that for the $s$ polarization, which is independent of the structure. This feature may be understood by the fact that better light confinement can be achieved for the $p$ polarized light where the $E$ field is not continuous across inhomogeneous material interfaces. For the $s$ polarization, the absorption of the strip structure becomes comparable to that of the nonstrip structure near grazing angles. The narrow absorption peaks due to the Wood's anomalies can improve the total absorption, but the improvement is smaller than that from the SPRs in the $p$-polarized light. For the $p$ polarization, the plasmoninduced absorption enhancement strongly "breaks" the limit of the generalized Lambert's cosine law and shows the "super-Lambertian" absorption. The broadband and wide-angle absorption enhancements by the SPRs will be of a great help for high-efficiency photovoltaic cells.

In conclusion, we have formulated the equation of the generalized Lambert's cosine law and comprehensively studied the $\mathrm{Au}$ nanostrip SC structure for enhancing the $s$ - and $p$-polarized light with detailed explanations of the enhancements by the Wood's anomalies and SPRs.

The authors acknowledge the support of grants 712108, 711508, and 711609 from the Research Grant Council of the Hong Kong Special Administrative Region, China. This project is also supported in part by a Hong Kong UGC Special Equipment Grant (SEG HKU09).

\section{References}

1. J. L. Balenzategui and F. Chenlo, Sol. Energy Mater. Sol. Cells 86, 53 (2005).

2. S. B. Rim, S. Zhao, S. R. Scully, M. D. McGehee, and P. Peumans, Appl. Phys. Lett. 91, 243501 (2007).

3. D. Cheyns, B. P. Rand, B. Verreet, J. Genoe, J. Poortmans, and P. Heremans, Appl. Phys. Lett. 92, 243310 (2008).

4. L. Dominici, L. Vesce, D. Colonna, F. Michelotti, T. M. Brown, A. Reale, and A. Di Carlo, Appl. Phys. Lett. 96, 103302 (2010).

5. H. A. Atwater and A. Polman, Nat. Mater. 9, 205 (2010).

6. H. P. Garg, Treatise on Solar Energy: Fundamentals of Solar Energy (Wiley, 1982).

7. R. A. Pala, J. White, E. Barnard, J. Liu, and M. L. Brongersma, Adv. Mater. 21, 3504 (2009).

8. C. J. Min, J. Li, G. Veronis, J. Y. Lee, S. H. Fan, and P. Peumans, Appl. Phys. Lett. 96, 133302 (2010).

9. A. Hessel and A. A. Oliner, Appl. Opt. 4, 1275 (1965).

10. M. G. Moharam, E. B. Grann, D. A. Pommet, and T. K. Gaylord, J. Opt. Soc. Am. A 12, 1068 (1995).

11. W. E. I. Sha, W. C. H. Choy, and W. C. Chew, Opt. Express 18, 5993 (2010).

12. A. D. Rakic, A. B. Djurisic, J. M. Elazar, and M. L. Majewski, Appl. Opt. 37, 5271 (1998).

13. W. C. H. Choy and H. H. Fong, J. Phys. D 41, 155109 (2008).

14. T. Tamir and S. Z. Zhang, J. Opt. Soc. Am. A 14, 1607 (1997). 